\title{
Emergence of macrostructures on mineral surfaces quantified with co- occurrence matrices
}

\author{
RICARDA D. ROHLFS ${ }^{1}$, INNA KURGANSKAYA ${ }^{1}$ AND \\ ANDREAS LUTTGE ${ }^{2}$
}

${ }^{1}$ Universität Bremen

${ }^{2}$ University of Bremen

Presenting Author: riccy@uni-bremen.de

Mineral dissolution processes are driven by atomic steps and stepwaves originating at lattice defects [1]. Trains of steps are advancing across the surface and are responsible for the resulting surface normal retreat. Etch pits that appear on the surfaces generate topographical diversity [2]. The development of complex 3D surface topographies are characterized by multifaceting and the formation of pits with steep walls [3]. The emergence of the so-called "macrostructures", such as macrosteps and other complex features, is caused by step bunching. Although step bunching was intensely studied for crystal growth processes [4] analogous studies for crystal dissolution are quite scarce. For example, the knowledge of the frequency and location of the step bunching events can help us to understand the mechanisms of macrostructure emergence.

Here, we used a new approach that is based on so-called cooccurrence matrices to collect statistical parameters on the step bunching that takes place on the calcite surfaces. This technique allows us to distinguish bunching events that occur at acute and obtuse atomic steps, and to collect statistics of the bunching for these two step types. We collected this information for synthetic data sets generated by our Kinetic Monte Carlo model of calcite dissolution that was developed previously [5], with the aim of learning the conditions under which the formation of macrofeatures begins. The new approach that we present here may be also applied to experimental data sets, e.g., the surface topographies obtained with atomic force microscopy.

[1] Lasaga \& Luttge (2001) Science, 2400-4.

[2] Fischer, Kurganskaya, Schäfer \& Lüttge (2014) Applied Geochemistry, 132-57.

[3] MacInnis \& Brantley (1992) Geochim. Cosmochim. Acta, 1113-26.

[4] Bredikhin (2008) Transport Theory and Statistical Physics, 504-19.

[5] Kurganskaya \& Luttge (2016) J. Phys. Chem. C, 6482-92. 